\title{
Acupoint herbal plaster for patients with primary dysmenorrhea: study protocol for a randomized controlled trial
}

Siyi Yu ${ }^{1 \dagger}$, Yueqiang Wen ${ }^{2 \dagger}$, Wanting $\mathrm{Xia}^{3+}{ }^{\dagger}$, Mingxiao Yang ${ }^{1}$, Zhengtao $\mathrm{Lv}^{4}$, Xiaoji Li ${ }^{1}$, Wenyao Li ${ }^{1}$, Sha Yang ${ }^{1}$, Youping $\mathrm{Hu}^{1}$, Fanrong Liang ${ }^{1 *}$ and Jie Yang ${ }^{1 *}$

\begin{abstract}
Background: Primary dysmenorrhea (PD), is one of main gynecological complaints in women of child-bearing age. Common medications for PD do not always achieve satisfactory outcome of pain relief. Hence, both health professionals and patients are seeking help from complementary and alternative medicine. The acupoint herbal plaster (AHP), which appears to be a safe and effective way to alleviate menstrual pain, as well as to improve other PD-related symptoms. Despite similar clinical studies for this condition in the past, no high-quality methodologybased clinical trial has been reported to date. The current study aims to assess the efficacy of the AHP compared with the acupoint placebo plaster (APP) and being placed on a waiting-list control group in patients with primary dysmenorrhea.
\end{abstract}

Methods/design: This study is a randomized, single-center, placebo-controlled clinical trial. A total of 180 women with PD will be included and randomly allocated to the AHP, APP and waiting-list (WL) groups in a 1:1:1 ratio. Patients in the AHP group will be provided with herbal plasters (Shaofuzhuyu decoction) on various acupoints: Shenque (CV8), Guanyuan (CV4), Qihai (CV5), Ciliao (BL32) and Zigong (EX-CA1). Women in the APP group will receive placebo plasters on the same acupoints, and no intervention will be given to the WL group until completion of the study. The primary outcome will be pain intensity reduction measured by a Visual Analog Scale (VAS), with other outcome measurements including the Cox Menstrual Symptom Scale (CMSS), the 12-Item Short Form Health Survey (SF-12) and the Participant Global Impression of Change (PGIC). All assessments will be performed at baseline, each menstrual cycle during the treatment course and the follow-up course. Any adverse events will be recorded throughout the study.

Discussion: This is the first study to compare the changes in menstrual pain after three different interventions: the active intervention (AHP), the placebo intervention (APP), and a period of no intervention (WL). This three-arm randomized controlled trial (RCT) aims to investigate the relative contributions of the specific (AHP vs. APP) and non-specific (APP vs. WL) effects to the overall clinical effects of the active AHP on women with PDM. The scientific and rigorous methodology design of this trial should gather good evidence to assess the curative effects and safety of the AHP on PD. Moreover, the results of this study may provide evidence-based references for the treatment of menstrual pain in future.

Trial registration: Chinese Clinical Trial Registry, ID: ChiCTR-TRC-16008701. Registered on 22 July 2016.

Keywords: Acupoint herbal plaster, Primary dysmenorrhea, Randomized controlled trial, Placebo control

\footnotetext{
*Correspondence: acuresearch@126.com; jenny_yang_jie@126.com

†'Siyi Yu, Yuegiang Wen and Wanting Xia contributed equally to this work.

${ }^{1}$ The Department of Acupuncture and Tuina, Chengdu University of

Traditional Chinese Medicine, Chengdu, Sichuan, China

Full list of author information is available at the end of the article
}

(c) The Author(s). 2019 Open Access This article is distributed under the terms of the Creative Commons Attribution 4.0 International License (http://creativecommons.org/licenses/by/4.0/), which permits unrestricted use, distribution, and reproduction in any medium, provided you give appropriate credit to the original author(s) and the source, provide a link to the Creative Commons license, and indicate if changes were made. The Creative Commons Public Domain Dedication waiver (http://creativecommons.org/publicdomain/zero/1.0/) applies to the data made available in this article, unless otherwise stated. 


\section{Background}

Primary dysmenorrhea (PD) is prevalent among adolescent girls and women of reproductive age [1]. The incidence of PD ranges from $45 \%$ to $72 \%$ of all menstruating women; moreover, among adolescent girls it can be as high as $93 \%$ [2, 3]. Its symptoms vary but typically include dull, throbbing and cramping pain in the lower abdomen during menstruation $[4,5]$. Patients may also experience vomiting, nausea, diarrhea, fatigue, fever, headache, sleeplessness and backache [6, 7]. This widespread condition not only decreases social participation and the quality of life of women, but also causes significant financial losses due to inability to work and extra medical costs $[8,9]$.

Approximately $67 \%$ of patients with PD choose anodyne drugs to temporarily relieve pain [10]. Nonsteroidal anti-inflammatory drugs (NSAIDs) are well advocated as the first-line medication for PD [11]. However, the pharmacological treatment has been reported to be associated with adverse effects including indigestion, headache, drowsiness; and in 20\%-25 of patients, failure to relieve the pain was reported [12]. Consequently, efforts have been made to identify effective, low-risk interventions $[13,14]$.

Acupuncture and moxibustion therapy has been used as a non-pharmacological analgesic method in China for hundreds of years. Some clinical studies have demonstrated that acupuncture and moxibustion can be as effective as, or even superior to, pharmacological drugs in relieving menstrual pain [15-17]. In addition, a number of systematic reviews and meta-analyses have also shown that acupuncture leads to overt reduction in the pain of dysmenorrhea $[18,19]$. As a typical external treatment in acupuncture, the acupoint herbal plaster (AHP) is a non-invasive, minimal-dose, transcutaneously absorbed, pharmacological treatment modality. Technically, its performance is based on the classical acupuncture and herbal medicinal theories. The treatment starts with placing processed Chinese herbal paste on a medical sticky plaster and then applying the plaster on certain acupoints. It is used for different treatment purposes in a much gentler format but can still achieve almost the same results of needling acupuncture. The AHP has been long known for its classical use of relieving respiratory conditions including pediatric recurrent pneumonia [20], stable chronic obstructive pulmonary disease [21], asthma [22] and rhinitis [23], as well as other conditions such as acute pancreatitis [24] and male infertility [25]. Nowadays, this traditional therapy has been innovated and developed to treat a much wider range of conditions including PD. Accordingly, modern clinical observational studies [26-28] have also indicated that the AHP has the potential to safely and effectively alleviate menstrual pain and improve PD-related symptoms.
According to the basic Traditional Chinese Medicine (TCM) theory, the major actions of the AHP are promoting blood circulation and moving energy $(Q i)$ within the human body. The underlying mechanism of the favorable effect of the AHP possibly originates from the dual effects of acupoint stimulation and drug actions. Based on different herbal formulas, the AHP has been reported to exert different therapeutic effects, including regulating cellular immune function, reducing pulmonary surfactant proteins, and reducing the severity of inflammation [29-32]. Modern pharmaceutical studies have demonstrated that paeoniflorin, petroleum ethersoluble fraction, eleven gingerols and others are the key chemical compound constituents within the herbs in the AHP [33-36]. The herbs that will be used in the plaster come from an ancient formula named "ShaoFu-ZhuYu Tang" (SFZYT).. This has been recognized as a safe and effective remedy to treat PD since the Qing Dynasty [37, 38]. An earlier pharmacological study showed that SFZYT inhibits the constriction of the uterine muscles and causes a favorable anti-inflammatory action [39]. Moreover, another study that employed PD-modeled rats indicated that the estradiol, oxytocin, progesterone, endothelin, $\beta$-endorphin and PGF2 $\alpha$ were restored to normal levels after intake of SFZYT [33]. These bioactive components within SFZYT can be absorbed transcutaneously from the herbal plaster. Hence, they can play a crucial role in regulating the whole body.

Unfortunately, there is a lack of high-quality clinical trials until now that have assessed the efficacy and safety of the AHP for PD. Therefore, we designed this clinical trial to fill this gap. To our knowledge, this study is the first RCT to date with a placebo design to test the efficacy and safety of the AHP on PD.

\section{Methods/design \\ Study design}

This proposed study is a single-centered, randomized, placebo-controlled trial which was devised following the Consolidated Standards of Reporting Trials (CONSORT) Statement recommendations [40] and the Standardized Protocol Items: Recommendations for Interventional Trials (SPIRIT) guidelines Additional file 1 [41]. Ethical approval has been obtained from the Institutional Review Board (IRB) of the Teaching Hospital of Chengdu University of Traditional Chinese Medicine (Approval No. 2016KL-003). We registered the study on the Chinese Clinical Trial Registry (Registration No. ChiCTR-IOR-16008701) in 2016.

The study will take place in the 3rd Affiliated Hospital of Chengdu University of TCM. During a run-in period of three menstrual cycles prior to randomization, the patients will be required to complete a Dysmenorrhea Diary (DD) and record related symptom scores every month to establish an individualized baseline score. A 
total of 180 subjects meeting the including criteria will be allocated randomly to three research groups that include one experimental group of the AHP and two control groups of the acupoint placebo plaster (APP) group and the waiting-list WL group. After randomization, subjects in the AHP and APP groups will receive treatments for another three menstrual cycles. Follow-up assessments through telephone calls or social media smartphone application will be processed for the last period of three menstrual cycles until the completion of study. Altogether it takes 9 months of three stages with three period cycles in each session. (See Fig. 1 for the study flowchart, and Fig. 2 for the assessment schedule).

\section{Participants}

\section{Recruitment strategies}

Study participants will be recruited mainly from the outpatient and inpatient sections of the Gynecology Department. Additionally, through flyers and social media advertisements, participants will be recruited from local communities and university campuses. All patients have the right to participate or drop out at any time, and will be required to sign the informed written consent before any study procedures commences.

\section{Inclusion criteria}

Participants who, in line with the following criteria, will qualify for the study are: (1) nulliparous women (1830 years old), (2) women who meet the diagnostic criteria of PD under the Primary Dysmenorrhea Consensus Guideline [11], (3) women who meet the TCM diagnosis of "qi and blood stagnation" or "cold and dampness retention," (4) women who have normal menstrual cycles (occurring every $28 \pm 7$ days), (5) a VAS score of the menstrual pain $\geq 40 \mathrm{~mm}$ during the baseline menstrual period, (6) women who agree to complete a DD during the study period, (7) women who agree to sign a informed consent form and volunteer for the research.

\section{Exclusion criteria}

Participants from the study who meet one or more following criteria will be excluded: (1) women with secondary dysmenorrhea caused by endometriosis, polycystic ovarian syndrome, pelvic inflammation and other gynecologic diseases, (2) women with any of the serious contraindications of cardiovascular, liver and kidney diseases, (3) women who are unable to express their subjective symptoms due to intellectual or mental conditions, (4) women with a malignant tumor, (5) women

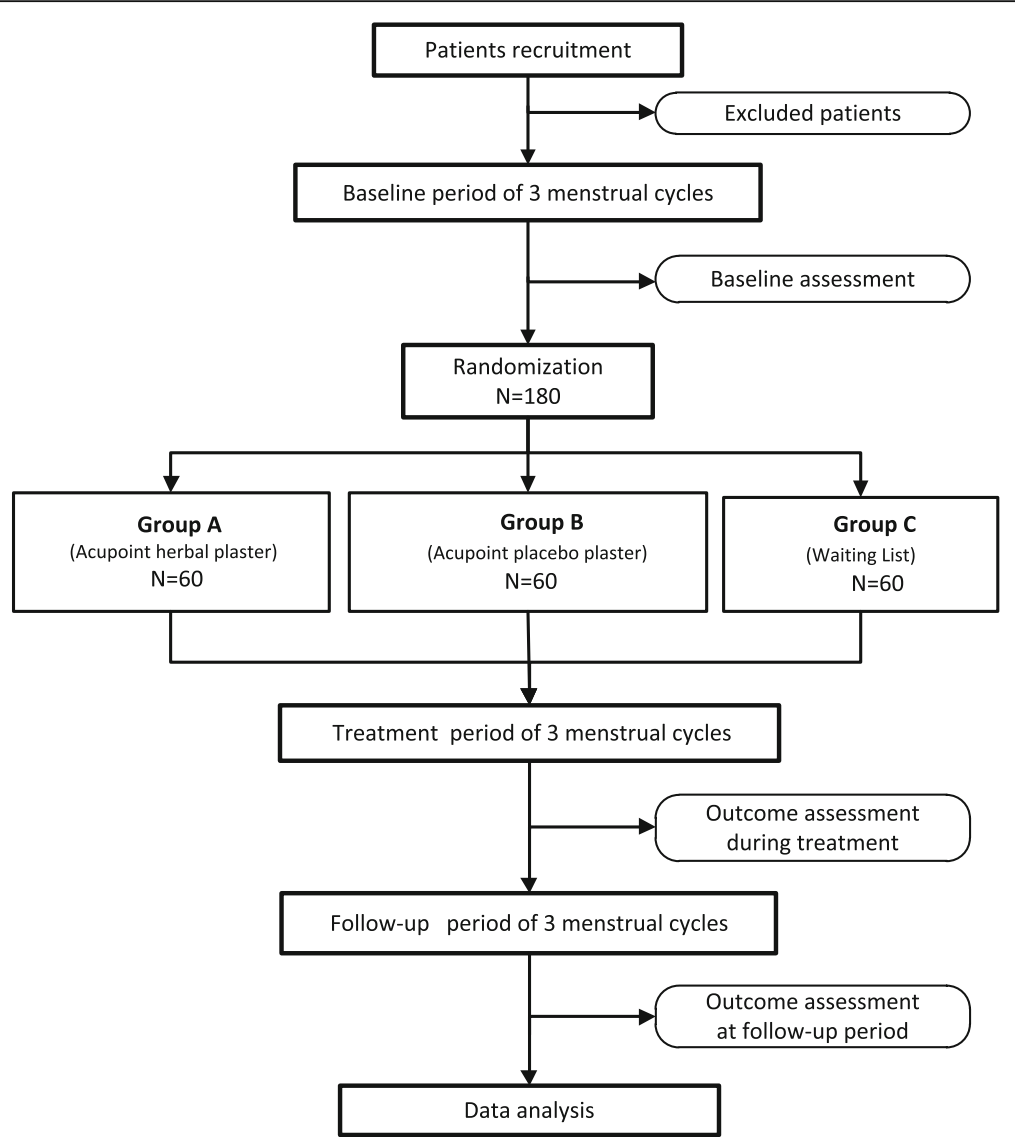

Fig. 1 Flowchart of the trial 


\begin{tabular}{|c|c|c|c|c|c|c|c|c|}
\hline & \multicolumn{8}{|c|}{ STUDY PERIOD } \\
\hline & \multirow{2}{*}{$\begin{array}{c}\text { Enrolment } \\
-3\end{array}$} & \multirow{2}{*}{$\frac{\text { Allocation }}{0}$} & \multicolumn{6}{|c|}{ Post-allocation } \\
\hline $\begin{array}{c}\text { TIMEPOINT } \\
\text { (menstrual cycle) }\end{array}$ & & & 1 & 2 & 3 & 4 & 5 & 6 \\
\hline \multicolumn{9}{|l|}{ ENROLMENT: } \\
\hline Eligibility screen & $\mathrm{X}$ & & & & & & & \\
\hline Informed consent & $\mathrm{x}$ & & & & & & & \\
\hline Demographics & $\mathrm{X}$ & & & & & & & \\
\hline Allocation & & $X$ & & & & & & \\
\hline \multicolumn{9}{|l|}{ INTERVENTIONS: } \\
\hline \multicolumn{9}{|l|}{$\begin{array}{c}\text { Group A } \\
\text { (acupoint herbal plaster) }\end{array}$} \\
\hline \multicolumn{8}{|l|}{$\begin{array}{c}\text { Group B } \\
\text { (acupoint placebo plaster) }\end{array}$} & \\
\hline \multicolumn{9}{|l|}{$\begin{array}{c}\text { Group C } \\
\text { (waiting list) }\end{array}$} \\
\hline \multirow{2}{*}{$\begin{array}{c}\text { ASSESSMENTS: } \\
\text { Gynecological abdominal } \\
\text { ultrasound B }\end{array}$} & & & & & & & & \\
\hline & $\mathrm{X}$ & & & & & & & \\
\hline VAS & $\mathrm{X}$ & & $\mathrm{X}$ & $\mathrm{X}$ & $\mathrm{X}$ & $\mathrm{X}$ & $\mathrm{X}$ & $\mathrm{X}$ \\
\hline CMSS & $\mathrm{X}$ & & $\mathrm{X}$ & $\mathrm{X}$ & $\mathrm{X}$ & $\mathrm{X}$ & $\mathrm{X}$ & $\mathrm{X}$ \\
\hline SF-12 & $\mathrm{X}$ & & $\mathrm{X}$ & $\mathrm{x}$ & $\mathrm{X}$ & $\mathrm{X}$ & $\mathrm{X}$ & $\mathrm{X}$ \\
\hline PGIC & $\mathrm{X}$ & & $\mathrm{x}$ & $\mathrm{X}$ & $\mathrm{X}$ & $\mathrm{X}$ & $\mathrm{X}$ & $\mathrm{X}$ \\
\hline \multirow[b]{2}{*}{ Dysmenorrhea diary } & $\mathrm{X}$ & & $\mathrm{X}$ & $\mathrm{X}$ & $\mathrm{X}$ & & & \\
\hline & $\mathrm{x}$ & & $\mathrm{X}$ & $\mathrm{x}$ & $\mathrm{X}$ & $\mathrm{X}$ & $\mathrm{X}$ & $\mathrm{X}$ \\
\hline Dysmenorrhea diary & & & $\mathrm{X}$ & $\mathrm{x}$ & $\mathrm{x}$ & $\mathrm{X}$ & $\mathrm{x}$ & $\mathrm{X}$ \\
\hline
\end{tabular}

Fig. 2 Standard Protocol Items: Recommendations for Interventional Trials (SPIRIT) Figure of enrollment, interventions and assessments. VAS Visual Analog Scale, CMSS Cox Menstrual Scale, SF-12 12-Item Short Form Health Survey, PGIC Participant Global Impression of Change, CEQ Credibility Expectancy Questionnaire

with poor compliance (i.e., cannot understand the purpose of this study or complete a DD during the baseline assessment), (6) pregnancy or breastfeeding, (7) women receiving another therapy or having received any other medical inventions for PD within the last 3 months.

\section{Randomization and allocation concealment}

After the screening visit, qualified subjects will be randomly assigned to either the AHP group, the APP group, or the waiting-list group in a ratio of $1: 1: 1$. Random numbers will be generated by a third person (BJ Gou) who is not practicing throughout the study, using the random number generator in the SPSS statistical software package (Version 22.0, SAS Institute Inc.). As the letters are drawn (A or B or C), they will be placed into opaque envelopes labeled with sequential numbers. The envelopes will be sealed and remain in numerical order in a safe place until the completion of surgery. The same researcher (BJ Guo) (not involved in the study) will prepare the envelopes. However, it was not possible to conceal the allocation among the waiting-list group.

\section{Blinding}

The herbal paste will be packed and labeled by the 3rd Affiliated Hospital of Chengdu University of TCM to ensure that the practitioner and the patients involved in the study will remain fully blinded as to the identity of the treatment administered. Therefore, the participants, clinical practitioners, the outcome evaluators, the data manager and statistician will not know the treatment allocations, which will not be revealed until the end of study. In addition, the practitioner will be instructed not to communicate with participants about the possibility 
of their allocation. However, blinding of the waiting-list group is not possible for this trial.

\section{Interventions}

Acupoint plaster treatment will be performed by certified acupuncturists with at least 5 years' clinical experience (Fig. 3a and b). All treatment details will be standardized between practitioners by guiding videos and relevant training before the first treatment session. The acupoint selection regimen was based on academic literature data mining [42] and expert opinions. Five acupoints will be used: Shenque (CV8), Guanyuan (CV4), Qihai (CV5), Ciliao (BL32) and Zigong (EX-CA1).

In this study, there are two types of paste to be applied on the sticky plasters: herbal paste (HP) for the AHP group, and buckwheat paste (BP) for the APP group (Fig. 3c and d). The HP remedy in this study comes from an ancient Chinese herbal formula named Shaofu Zhuyu Tang (SFZYT) which was recorded in a TCM medical classic, Yi Lin Gai Cuo (Correction of Errors in Medical Classics), which was written or recorded by Qingren
Wang in 1830. Clinical studies have shown the positive effect of SFZYT on PD [43, 44]. A systematic review recently found the therapeutic effects of SFZYT on PD to be superior to drugs [45]. In addition, previous clinical observations [46-48] have demonstrated that the SFZYT was safe for acupoint plaster. Therefore, we adapted this formula and standardized its performance.

In the AHP group, the standard operating procedures (SOP) are listed as follows:

1. Prepare the herbal materials: SFZYT includes Angelicae sinensis Radix (danggui), Rhizoma (chuanxiong), Paeoniae Radix rubra (chishao), Cinnamomi cortex (rougui), Foeniculi fructus (xiaohuixiang), Zingiberis rhizoma (ganjiang), Myrrha (moyao), Trogopterus dung (wulingzhi), Typhae Pollen (puhuang) and Corydalis rhizoma (yanhusuo). The ingredients of this formula are mixed in a weight ratio of $3: 1: 2: 1: 0.5: 2: 1: 3: 1: 1$ and then the raw materials ground into fine powder (under $75 \mu \mathrm{m}$ diameter) using a special Chinese

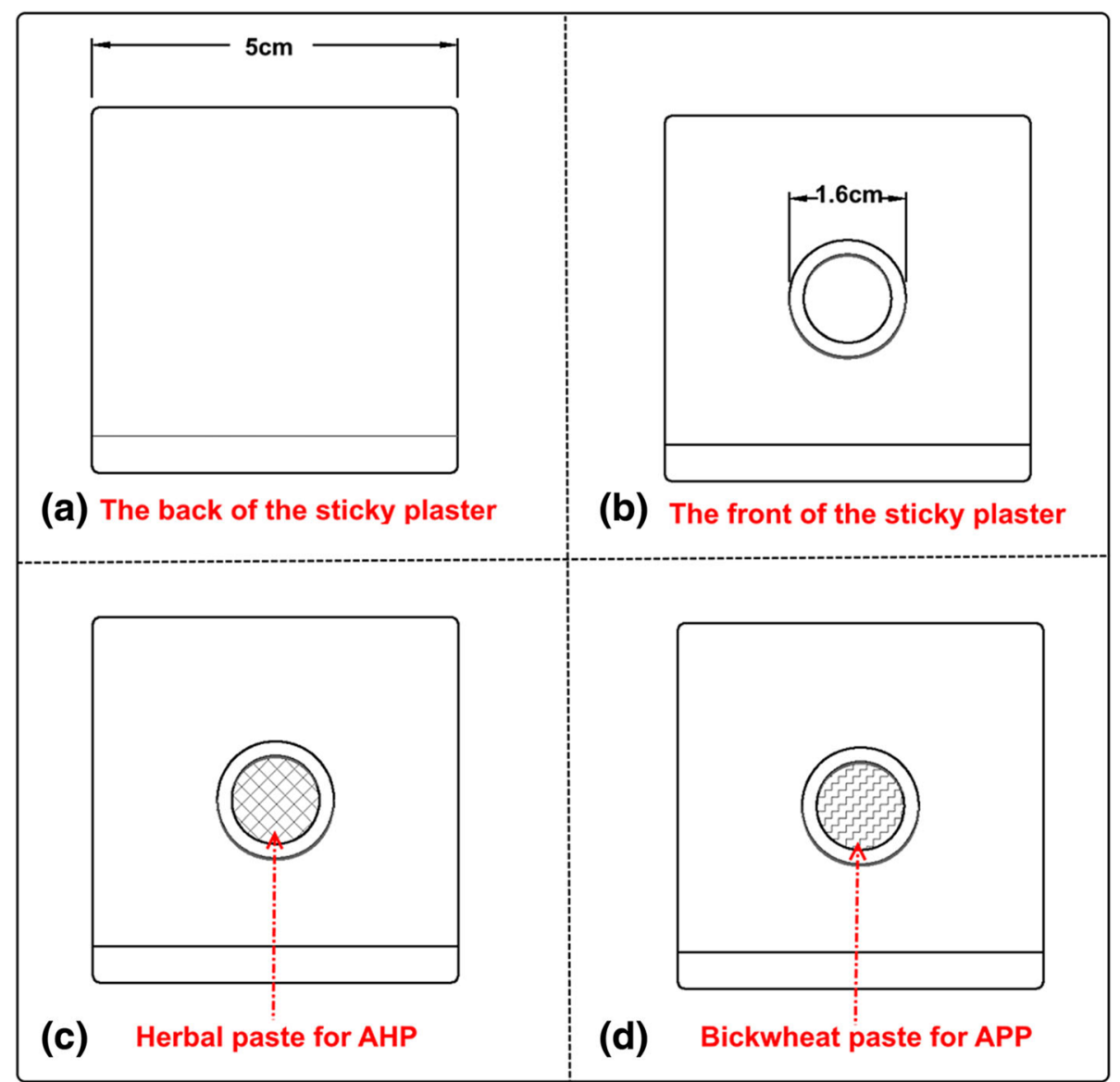

Fig. 3 Illustration of the sticky plaster. $\mathbf{a}$ and $\mathbf{b}$ show the anterior and posterior appearance of the acupoint plaster, respectively. c and $\mathbf{d}$ show the paste inside the plaster. $\mathbf{c}$ demonstrates the herbal plaster. $\mathbf{d}$ demonstrates the bluckwheat plaster. These two kinds of paste are indentical in appearance. The Figs. are only for illustrative purposes 
medicinal crop powder mixer (manufactured by Royalstar Co., Ltd., Hefei, China)

2. Add the liquid base: place the mixed herbal powder into a container, add honey and stir well to make a thick herbal paste

3. Add the sticky plaster: put $10 \mathrm{~g}$ of the herbal paste onto a medical sticky plaster. Then apply the plaster over the acupoints. The sticky plasters are sized $5 \times 5 \mathrm{~cm}^{2}$ with a $0.8-\mathrm{cm}$ radius in the middle of which is placed the host herbal paste. The rest of the sticky plaster carries no herbal paste, which makes it adhere to the skin over the acupoints

4. Treatment procedures: locate the acupoints, disinfect the skin, and attach the plaster on the skin over the acupoints. The plasters will be left on the skin for 4-8 h depending on each patient's individual response because it must be removed immediately should any allergic reaction occur, which may be described by patients as a burning sensation, severe itchiness, blisters, pruritus or rash on the local skin. A total of nine sessions will be performed during the three menstrual cycles, the treatment starts 1 week prior to the menstruation, with one 6-8-h treatment three times per week, according to the subject's estimated dates

For the APP group, the placebo paste is made of buckwheat flour without any herbal medicines; however is still mixed with honey to maintain a similar appearance to the AHP (Fig. 2c and d) so that the plasters used in these two experimental groups are undistinguishable to patients and practitioners. Moreover, the only variable between the AHP group and the APP group is the ingredients of paste. In order to reduce the bias caused by performance, all the rest of the clinical procedures remain the same.

No intervention will be performed in the WL group. The participants are required to continue their current living habits, including exercise and diet, while filling out related questionnaires throughout the full observational period of six menstrual cycles. To meet the requirements of the Rule of Ethics, all participant in the WL group will be offered free AHP treatment of their choice at the completion of the study.

With regard to quality control of the herbal plaster, we will take the following measures: (1) first, the herbal plaster will be prepared beforehand by the central pharmacy of the 3rd Affiliated Hospital of Chengdu University of TCM that meets the requirements of the regulatory guidance issued by the China Food and Drug Administration, (2) in addition, pre-job SOP training and examination will be provided by the principal investigator (Prof. J Yang) to the practitioner for ensuring the quality of treatment procedures (i.e., locate the acupoints, disinfect the skin, attach the plaster on the skin over acupoints), (3) furthermore, the data and results will be monitored by the Evidence-based Medicine Center of Chengdu University of Traditional Chinese Medicine.

\section{Outcome measurements \\ Primary outcome}

The primary outcome will be pain intensity reduction measured by the Visual Analog Scale (VAS). The severity of menstrual pain will be estimated using the VAS graded from $0 \mathrm{~mm}$ to $100 \mathrm{~mm}$, indicating an absence of pain to the most serious pain imaginable [49].

\section{Secondary outcomes}

The secondary outcomes include the Cox Menstrual Symptom Scale (CMSS) [50], the 12-Item Short Form Health Survey (SF-12) [51], the Participant Global Impression of Change (PGIC) [52] and the DD.

\section{Time points of outcome measurements}

The VAS, CMSS, SF-12 and DD will be recorded at baseline and at every menstrual cycle throughout the treatment and the follow-up periods. The PGIC will be measured at the first, second, third, fourth, fifth and sixth menstrual cycles after inclusion.

We will estimate the expectation of participants to determine whether their expectations affected the outcomes by using the Credibility Expectancy Questionnaire (CEQ) [53, 54] at the baseline and the first to the third menstrual cycle before receiving treatment. In the proposed study, the questionnaire will be used to control for placebo effects, which are expected to be relatively high for light-therapy studies [54]. Blinding will be assessed by asking participants what group they thought they were in (AHP group or APP group, or "don't know") at the end of trial, and why they believed that they were in that group.

\section{Safety evaluation}

Clinical adverse events (AEs) will be recorded throughout the study and graded using the National Cancer Institute Common Terminology Criteria for Adverse Events (CTCAE) version 4 [55]. According to previous randomized controlled trials (RCTs) [56, 57], the AHP may cause potential adverse events of itchiness, allergic responses of the skin or the whole body, local infections and other mild side effects. Any unexpected symptoms or signs during the treatment must be documented regardless of their relation to the study intervention. All details of related and unexpected AEs, such as time of occurrence, severity of $\mathrm{AE}$, and suspected causes, will be recorded on Case Report Forms (CRFs). Participants with mild and moderate AEs will receive symptomatic treatment and will be closely followed up by the research team. Severe AEs will be reported to the Research Ethics Committee within $48 \mathrm{~h}$. 


\section{Sample size calculation}

A few scientific trials have investigated the biomechanical effects of acupoint plaster on PD; however, the research methodologies are not appropriate to reference the determination of sample size to this study. The article being used for our study, was from an acupuncture clinical report with the use of VAS scores [16]. Liu's study reported that patients who received classical acupoint achieved a mean reduction of 36.1 in VAS after acupuncture; while patients who received non-acupoint treatment showed a worse score of 29.9. Therefore, the decrease in VAS score of the three groups was predicted to be 38,29 and 26, respectively. Using a bilateral test with an alpha value of $5 \%$ and a power of $80 \%$, standard deviation for each group was $20 \mathrm{~mm}$. According to the calculation with PASS software (Version 11.0, NCSS, LLC. Kaysville, UT, USA) in a 1:1:1 ratio, the effect size is 0.2550 of 51 participants in each group. The sample size is also calculated using the following formula [58]:

$$
n=2\left(\sigma \frac{z_{1-\alpha /(2 \tau)}+z_{1-\beta}}{\mu_{A}-\mu_{B}}\right)^{2} .
$$

Allowing for a $15 \%$ dropout, the sample size was increased to 60 per group, making a total of 180 .

\section{Statistical analysis plan \\ Data integrity}

All participants are assessed at run-in period and at the first, second, third, fourth, fifth and sixth menstrual cycles after recruitment. A well-trained assessor, who is blinded to the treatment assignment, will collect clinical data using CRF files at each visit. Another two pretrained data managers will verify and cross-check the CRFs, for the sake of ensuring reliability and accuracy of the data. If there are any queries in the CRFs, the results will be sent to the third investigator for resolution. Data managers will also be responsible for administration, coordination and monitoring (including Excel spread sheet-set up, data entry, coding and query management). Any incomplete data will be coded as unknown, missing or not applicable, and all data will be anonymously extracted to keep the identities of patients confidential. Results of the analysis must not be released with individual identification of the subject until the Excel spread sheet is closed.

\section{Methods of statistical analyses}

Data analyses will be processed with SPSS 22.0 (SPSS Inc., Chicago, IL, USA) and supervised by a skilled statistician blinded to group allocation.

The Full Analysis Set (FAS) is for all participants who have been randomized in terms of the intention-to-treat (ITT), and the per-protocol analysis set (PPS) and is for the individuals who complete the trial and do not have significant protocol violations. On the basis of rule of the last-observation-carried-forward, missing values will be imputed. In order to evaluate therapeutic effect and safety, the FAS and PPS will be used simultaneously.

Categorical data, such as gender and medical history, will be tabulated with frequencies or percentages; and continuous data, such as age and disease course, will be reported as mean \pm standard deviation (SD), or median. For the baseline variables, sociodemographic data and other basic indicators will be carried out using analysis of variance (ANOVA) and $\chi^{2}$. To compare variables before and after treatment in the same group, a paired $t$ test will be used. Repeated measures analysis of variance (ANOVA) will be used to compare the intergroup differences among the three groups. Tests of ITT between the AHP and APP arms and between the APP and WL arms with respect to the change will based on timeintervention interactions in the mixed-effect models. A $P$ value of less than 0.05 (two-sided) indicates a statistically significant difference, with $95 \%$ confidence intervals.

\section{Discussion}

Currently, there have been no methodologically controlled clinical studies confirming the efficacy of AHP therapy. To meet the demand for high-quality RCTs, our team has designed this randomized placebo-controlled trial to explore the potential therapeutic effect of the AHP for PD. In this pilot study, we will compare the change in menstrual pain after administration of three interventions: the active intervention (AHP), the placebo intervention (APP), and a period of no intervention (WL). This three-arm RCT aims to investigate the relative contributions of the specific (AHP vs. APP) and the non-specific (APP vs. WL) effects to the overall clinical effects of the AHP on women with PDM. In particular, a growing body of studies $[58,59]$ has confirmed that the sham control may play a crucial part in evaluating the effectiveness of a treatment, which may help separate the specific and non-specific effects. Therefore, the active and sham acupoint plasters should be adopted to investigate the specific analgesic effect on patients with PDM. In order to minimize the placebo effect, buckwheat flour paste is used in the placebo arm, which may successfully blind the patients and the practitioners due to their identical appearance. For the purpose of comparing the non-specific (sham intervention vs. no treatment) effects and avoiding bias caused by psychological influences, we set the third group of the waiting-list control without any acupuncture interventions. This is because participants in the APP group may have a placebo effect due to the unawareness of group allocation and the treatment that they are undergoing; therefore, 
placebo effects may happen secondary to their strong beliefs of being in the AHP group.

To our knowledge, the present study will be the first clinical trial that compares the AHP with the APP, and being placed in a waiting-list control group in treating PD. The scientific and rigorous methodologic design of this trial hopefully will provide consolidated evidence on the efficacy and safety of the AHP for treating $\mathrm{PD}$, and hopefully provide reference to clinical practice Additional file 2.

\section{Trial status}

Participant recruitment is currently ongoing.

\section{Additional files}

Additional file 1: Standard Protocol Items: Recommendations for Interventional Trials (SPIRIT) 2013 Checklist: recommended items to address in a clinical trial protocol and related documents. (DOC $123 \mathrm{~kb}$ )

Additional file 2: Standards for Reporting Interventions in Clinical Trials of Acupuncture (STRICTA). (DOCX $17 \mathrm{~kb}$ )

\section{Abbreviations}

AEs: Adverse events; AHP: Acupoint herbal plaster; ANOVA: Analysis of variance; AP: Acupoint plaster; APP: Acupoint placebo plaster; BP: Buckwheat plaster; CMSS: Cox Menstrual Symptom Scale; CONSORT: Consolidated Standards of Reporting Trials; CRF: Case Report Form; DD: Dysmenorrhea Diary; FAS: Full analysis set; HP: Herbal plaster; IRB: Institutional Review Board; ITT: Intention-to-treat; NSAIDs: Nonsteroidal anti-inflammatory drugs; PD: Primary dysmenorrhea; PGIC: Participant Global Impression of Change; PPS: Per-protocol analysis set; RCT: Randomized controlled trial; SD: Standard deviation; SF-12: 12-Item Short Form Health Survey; SFZYT: Shaofu-Zhuyu Tang; SOP: Standard operating procedures; TCM: Traditional Chinese Medicine; VAS: Visual Analog Scale; WL: Waiting list

\section{Acknowledgements}

The authors thank all of the collaborators and participants of the study.

\section{Funding}

This trial is supported by the program of People Benefit Project of the Technology Bureau of Chengdu (Grant No. 2015-HM01-00195-SF), the program of Fok Ying-Tong Education Foundation for Young Teachers in the Higher Education Institution of China (Grant No. 20155132210002) and the programs of the National Natural Science Foundation of China (Grant Nos. 81303060 and 81574089).

\section{Availability of data and materials}

All data and material supporting our findings can be obtained from the main author.

\section{Authors' contributions}

JY and FL contributed to the design of the study protocol. SY, YW and WX are directors of the recruitment sites, provided clinical expertise and drafted the study protocol. MY, ZL, XL, WL and YH critically revised the manuscript and will conduct the study. All authors have read and approved the final manuscript.

\section{Ethics approval and consent to participate}

This study protocol has been approved by the Institutional Review Board of the Teaching Hospital of Chengdu University of TCM (Approval No. 2016KL003). Any modifications to the research protocol will be notified to this Human Research Ethics Committee. Informed consent will be obtained from each subject prior to enrollment. The study conforms to the principles of the Declaration of Helsinki and Good Clinical Practice guidelines.

\section{Consent for publication}

No personal data will be published, and the informed consent form will include permission to publish group data. Results will be disseminated in peer-reviewed publications and published in international journals.

\section{Competing interests}

The authors declare that they have no competing interests.

\section{Publisher's Note}

Springer Nature remains neutral with regard to jurisdictional claims in published maps and institutional affiliations.

\section{Author details}

${ }^{1}$ The Department of Acupuncture and Tuina, Chengdu University of Traditional Chinese Medicine, Chengdu, Sichuan, China. ${ }^{2}$ The Department of Basic Medical Sciences, Chengdu University of Traditional Chinese Medicine, Chengdu, Sichuan, China. ${ }^{3}$ The Department of Clinical Medical, Chengdu University of Traditional Chinese Medicine, Chengdu, Sichuan, China. ${ }^{4}$ Department of Orthopedics, Tongji Hospital, Tongji Medical College, Huazhong University of Science and Technology, Wuhan, China.

Received: 19 April 2017 Accepted: 10 May 2018

Published online: 03 July 2018

\section{References}

1. Kennedy S. Primary dysmenorrhoea. Lancet. 1979:56:1116.

2. Yang J, Chen J, Lao L, Yang M, Chen J, Bo L. Effectiveness study of moxibustion on pain relief in primary dysmenorrhea: study protocol of a randomized controlled trial. Evid Based Complement Alternat Med. 2014; 2014:1-6.

3. Ju H, Jones M, Mishra G. The prevalence and risk factors of dysmenorrhea Epidemiol Rev. 2014;36:104-13

4. Grandi G, Ferrari S, Xholli A, Cannoletta M, Palma F, Romani C, Volpe A, Cagnacci A. Prevalence of menstrual pain in young women: what is dysmenorrhea? J Pain Res. 2012;2012:169-74.

5. Zahradnik HP, Groth BK. Nonsteroidal anti-inflammatory drugs and hormonal contraceptives for pain relief from dysmenorrhea: a review. Contraception. 2010;81:185-96.

6. Harel Z. Dysmenorrhea in adolescents. Menarche Transit Girl Woman. 2008; 1135:185-95.

7. lacovides S, Avidon I, Baker FC. What we know about primary dysmenorrhea today: a critical review. Hum Reprod Update. 2015;21:17.

8. Faramarzi M, Salmalian H. Association of psychologic and nonpsychologic factors with primary dysmenorrhea. Iran Red Crescent Med J L. 2014; https://www.doi.org/10.5812/ircmj.16307.

9. Ruoff $G$, Lema M. Strategies in pain management: new and potential indications for COX-2 specific inhibitors. J Pain Symptom Manag. 2003;25:21-31.

10. Jun E-M, Chang S, Kang D-H, Kim S. Effects of acupressure on dysmenorrhea and skin temperature changes in college students: a non-randomized controlled trial. Int J Nurs Stud. 2007:44:973-81.

11. Lefebvre G, Pinsonneault O, Antao V, Black A, Burnett M, Feldman K, Lea R, Robert M. Primary dysmenorrhea consensus guideline. J Obstet Gynaecol Can. 2005:27:1117

12. Park J-S, Park S, Cheon $\mathrm{C}-\mathrm{H}, \mathrm{Go} H-\mathrm{Y}$, Sun $\mathrm{S}-\mathrm{H}$, Shin $\mathrm{Y}-\mathrm{C}$, Jang $\mathrm{B}-\mathrm{H}, \mathrm{Ko}$ S-G Effects of Gyejibongnyeong-hwan on dysmenorrhea caused by blood stagnation: study protocol for a randomized controlled trial. Trials. 2012;13:6.

13. Proctor M, Hing W, Johnson TC, Murphy PA, Brown J. Spinal manipulation for dysmenorrhoea. Cochrane Database Syst Rev. 2006; https://www.doi. org/10.1002/14651858.CD002119.

14. Gou CQ, Gao J, Wu CX, Bai DX, Mou HY, Hou XL, Zhao X. Moxibustion for primary dysmenorrhea at different interventional times: a systematic review and meta-analysis. Evid Based Complement Alternat Med. 2016; https:// www.doi.org/10.1155/2016/6706901.

15. Sriprasert I, Suerungruang S, Athilarp P, Matanasarawoot A, Teekachunhatean S. Efficacy of acupuncture versus combined oral contraceptive pill in treatment of moderate-to-severe dysmenorrhea: a randomized controlled trial. Evid Based Complement Alternat Med. 2015; https://www.doi.org/10.1155/2015/735690.

16. Liu CZ, Xie JP, Wang LP, Liu YQ, Song JS, Chen YY, Shi GX, Zhou W, Gao SZ, Li SL. A randomized controlled trial of single point acupuncture in primary dysmenorrhea. Pain Med. 2014;15:910-20. 
17. Yang $M$, Chen X, Bo L, Lao L, Chen J, Yu S, Yu Z, Tang H, Yi L, Wu X. Moxibustion for pain relief in patients with primary dysmenorrhea: a randomized controlled trial. PLoS One. 2017;12:17.

18. Smith CA, Zhu X, He L, Song J. Acupuncture for primary dysmenorrhoea. Cochrane Database Syst Rev. 2011:40:132.

19. Cho SH, Hwang EW. Acupuncture for primary dysmenorrhoea: a systematic review. BJOG Int J Obstet Gynaecol. 2010;117:509-21.

20. Wang L, Pang L, Bai X, Zhao L, Liu F, Lv X. Clinical efficacy on pediatric recurrent pneumonia treated with point application in summer for the prevention in winter. Chin Acupunct Moxibust. 2016;36:261.

21. Li JS, Li SY, Yu XQ, Xie Y, Wang MH, Li ZG, Zhang NZ, Shao SJ, Zhang YJ, Zhu L. Bu-Fei Yi-Shen granule combined with acupoint sticking therapy in patients with stable chronic obstructive pulmonary disease: a randomized, double-blind, double-dummy, active-controlled, 4-center study. J Ethnopharmacol. 2012;141:584-91.

22. Lai X, Li Y, Fan Z, Zhang J, Liu B. An analysis of therapeutic effect of drug acupoint application in 209 cases of allergic asthma. J Tradit Chin Med. 2001;21:122-6.

23. Peng J, Wu X, Hu J, Fang Y, Zi M, Liu B. Influencing factors on efficacy of summer acupoint application treatment for allergic rhinitis: a retrospective study. J Tradit Chin Med. 2012;32:377-81.

24. Ge H, Chen B. Clinical observation of acute pancreatitis treated with acupoint application combined with medicine. Chin Acupunct Moxibust. 2012;32:602-4

25. Peng S, Zheng Y, Zheng K, Lin K, Wu J, Zheng W, Li Y. Effect of a comprehensive therapy plus Gushenyutai plaster administered at Guanyuan (CV 4) on male infertility associated with semen non-liquefaction. J Tradit Chin Med. 2014;34:666-72.

26. Wang S, Lu D, Li Y. Observation on therapeutic effect of acupoint application on dysmenorrhea of excess syndrome and effect on prostaglandins. Chin Acupunct Moxibust. 2009;29:265-8.

27. Feng $X, L i X$, Huang $Y$. Observation on 60 cases of primary dysmenorrheal treated with acupoint-application. Liaoning J Tradit Chin Med. 2013:6:1225-6.

28. Yang Y. Observation on primary dysmenorrhea treated with acupointapplication. Hubei J Tradit Chin Med. 2017:1:32-3.

29. Liu LY, Qiao M, Gao F, Wang HS, Sun JH. Effect of acupiont sticking therapy with Kechuanting paste on expression of T-bet and GATA-3 mRNA in lung for chronic asthma airway inflammation rats. J Nanjing Univ Tradit Chin Med. 2014;6:550-3.

30. Chen J, Chen JQ, Huang Y, Zhang JL, Tang CZ. Study of traditional Chinese medicine acupoint sticking ultrafine effect on the TGF- $\beta 1$ and IL-17 content in allergic rhinitis rats. Chin J Basic Med Trad Chin Med. 2014;9:1218-21.

31. Ding $Y$, Wang $Y$. The effects of acupoint sticking therapy in summer for winter disease on inflammation mediator in patients with chronic bronchitis. Int J Laboratory Med. 2014;17:2289-90.

32. Yao $\mathrm{H}$, Tong J, Zhang $\mathrm{P}$, Wang Z. Effect of acupoint application on inflammatory cells level in patients with bronchial asthma. J Guangzhou Univ Tradit Chin Med. 2007:4:289-92.

33. Huang X, Su S, Duan J, Sha X, Zhu KY, Guo J, Yu L, Liu P, Shang E, Qian D. Effects and mechanisms of Shaofu-Zhuyu decoction and its major bioactive component for Cold - Stagnation and Blood - Stasis primary dysmenorrhea rats. J Ethnopharmacol. 2016;186:234-43.

34. Wang L, Huang S, Chen B, Zang XY, Su D, Liang J, Xu F, Liu GX, Shang MY, Cai SQ. Characterization of the anticoagulative constituents of Angelicae Sinensis Radix and their metabolites in rats by HPLC-DAD-ESI-IT-TOF-MSn. Planta Med. 2016:82:362-70.

35. Xin Y, Liu S. Quantitative assessment of the influence of Rhizoma Zingiberis on the Level of aconitine in rat gut sacs and qualitative analysis of the major influencing components of Rhizoma Zingiberis on aconitine using UPLC/MS. PLoS One. 2015;10:16

36. Tian Y, Li J, Li Y, Dong Y, Yao F, Mao J, Li L, Wang L, Luo S, Wang M. Effects of Bufei Yishen granules combined with acupoint sticking therapy on pulmonary surfactant proteins in chronic obstructive pulmonary disease rats. Biomed Res Int. 2016;2016:1-8

37. Fan M. Clinical observation of Shaofu Zhuyu decotiong for treating primary dysmenorrhea of cold coagulation and blood stasis. Beijing J Tradit Chin Med. 2011;30:455-6.

38. Cheng Q, Zhu Y. Clinical study on primary dysmenorrheal of blood-stasis of cold-coagulation syndrome treated with modified Shaofu Zhuyu decoction. J Chin Med. 2011;26:1249-50.
39. Ye X, Wang H, Le J. Effects of Shaofuzhuyu Tang on uterine convulsion and anti-inflammatory action in rodent. Chin J Hosp Pharm. 2002:22:329-31.

40. Schulz KF, Altman DG, Moher D. CONSORT 2010 statement: updated quidelines for reporting parallel group randomized trials. BMC Med. 2010;8:18.

41. Chan AW, Tetzlaff JM, Altman DG, Laupacis A, Gøtzsche PC, Krležajerić K, Hróbjartsson A, Mann H, Dickersin K, Berlin JA. SPIRIT 2013 Statement: Defining Standard Protocol Items for Clinical Trials. Ann Intern Med. 2013; 158:200-7.

42. Yu S, Yang J, Yang M, Gao Y, Chen J, Ren Y, Zhang L, Chen L, Liang F, Hu Y. Application of acupoints and meridians for the treatment of primary dysmenorrhea: a data mining-based literature study. Evid Based Complement Alternat Med. 2015; https://www.doi.org/10.1155/2015/752194.

43. Zhang X, Zhang S, Xu C. Jiawei Shaofu Zhuyu decoction combined with moxibustion therapy in treating primary dysmenorrhea - 55 cases. Chin J Exp Tradit Med Formulae. 2014;20:192-5.

44. Wang N, Li P. Analysis of therapeutic effects of modified Shaofu Zhuyu decoction for dysmenorrhea caused by coagulation and blood stasis. J Clin Med Pract. 2013;5:036

45. Lee H, Choi T-Y, Myung C-S, Lee JA, Lee MS. Herbal medicine (Shaofu Zhuyu Decoction) for treating primary dysmenorrhea: a systematic review of randomized clinical trials. Maturitas. 2016:86:64-73.

46. Lin D. The acupoint application of Shaofu Zhuyu Tang on navel. J Extern Ther TCM. 2000;9:36-7.

47. Li J, Zhang M, Wang $X$. The clinical observation on the treatment of chronic pelvic inflammatory disease by oral and external application of Shaofu Zhuyu Tang. J Qinghai Med. 2014;44:53-4.

48. Fu J, Xia Y. The clinical observation on oral and external application of Shao Xue Tong Yu decoctionon for treating chronic pelvic inflammatory disease. Mod J Integrat Trad Chin Western Med. 2012;21:1513-4.

49. Price DD, Mcgrath PA, Rafii A, Buckingham B. The validation of visual analogue scales as ratio scale measures for chronic and experimental pain. Pain. 1983;17:45-56.

50. Cox DJ, Meyer RG. Behavioral treatment parameters with primary dysmenorrhea. J Behav Med. 1978;1:297-310.

51. Bourion-Bédès $S$, Schwan $R$, Laprevote $V$, Bédès $A$, Bonnet $J$, Baumann $C$. Differential item functioning (DIF) of SF-12 and Q-LES-Q-SF items among French substance users. Health Qual Life Outcomes. 2015;13:1-8.

52. Pagé I, Abboud J, O Shaughnessy J, Laurencelle L, Descarreaux M. Chronic low back pain clinical outcomes present higher associations with the STarT Back Screening Tool than with physiologic measures: a 12-month cohort study. BMC Musculoskelet Disord. 2015;16:1-10.

53. Smeets RJ, Beelen S, Goossens ME, Schouten EG, Knottnerus JA, Vlaeyen JW Treatment expectancy and credibility are associated with the outcome of both physical and cognitive-behavioral treatment in chronic low back pain. Clin J Pain. 2008:24(4):305-15.

54. Devilly GJ, Borkovec TD. Psychometric properties of the credibility/ expectancy questionnaire. J Behav Ther Exp Psychiatry. 2000;31(2):73-86.

55. US Department of Health and Human Services. Common terminology criteria for adverse events (CTCAE) version 4.0[J]. National Cancer Institute. 2009;(09-5410).

56. Wu X, Jin P, Li G, Wei Z, Liu G, Liu B. Efficacy evaluation of summer acupoint application treatment on asthma patients: a two-year follow-up clinical study. J Tradit Chin Med. 2015:35:21-7.

57. Wu XQ, Peng J, Li GQ, Su HP, Liu GX, Liu BY. Association between skin reactions and efficacy of summer acupoint application treatment on chronic pulmonary disease: A prospective study. Chin J Integr Med. 2016;22 284-92.

58. Chow SC, Shao J, Wang H. Sample size calculations in clinical research. Boca Raton: Chapman \& Hall/CRC Biostatistics; 2003.

59. Irnich D, Salih N, Offenbächer M, Fleckenstein J. Is sham laser a valid control for acupuncture trials? Evid-Based Complement Altern Med. 2011;2011(27): 485945. 